\title{
Renal FMH with AV fistula
}

\begin{abstract}
Renal Fibro Muscular Hyperplasia (FMH) is an abnormal condition which causes a "banding" or "string of pearls" effect of the the arterial wall. This "beading" typically results in stenosis of the renal arteries, but may also be present within the carotid arteries or other peripheral arteries. FHH is often associated with AV fistulas, where the artery and vein have an abnormal connection. FMH is seen predominately in the female population whom present with uncontrolled hypertension. The etiology is suspected to be genetic, but confirmation has not been established. The diagnosis of FMH must be confirmed with CTA, MRA or conventional angiographical correlation. This case study demonstrates an unforeseen finding of an AV fistula with CTA and angiogram and the correlative of doppler waveforms seen with renal artery stenosis.
\end{abstract}

Keywords: fibro muscular hyperplasia, renal artery stenosis, hypertension, av fistula
Volume 3 Issue 5 - 2015

\section{Don R Peterson}

Diagnostic sonographer, Pasco Cardiology Center, USA

Correspondence: Don R Peterson, Diagnostic sonographer, Pasco Cardiology Center, 3633 Little Road, Suite I0I New Port Richey, FL 34655, Florida, USA, Tel (727) 372-5952,

Email donrobpete@yahoo.com

Received: February 26, 2015 | Published: October 12, 2015

\section{Case report}

A 71year old female with chronic hypertension and remote history of fibro muscular hyperplasia $(\mathrm{FMH})$ of the renal arteries was evaluated by our cardiology group. Despite being prescribed multiple anti-hypertensive medications, her blood pressure remained sub optimally controlled. Her systolic pressures were documented at $150-160 \mathrm{~mm} \mathrm{Hg}$ with diastolic components as high as $100-110 \mathrm{~mm}$ $\mathrm{Hg}$. Approximately 15years ago, the patient underwent bilateral renal artery balloon angioplasties. In view of the above clinical findings, a bilateral renal artery doppler was requested. ${ }^{1}$

The examination was performed by a registered vascular technologist on a GE Vivid-q ultrasound unit with a M4-S transducer. Gray scale, color and doppler images were obtained from the mid abdominal aorta, the right renal artery and left renal artery. Upon interrogation of the right renal artery, markedly abnormal waveforms and velocities were demonstrated throughout the entire length of the vessel. ${ }^{2}$ The peak systolic velocity (PSV) was measured at $351 \mathrm{~cm} /$ $\mathrm{sec}$ with an elevated end diastolic velocity of $140 \mathrm{~cm} / \mathrm{sec}$. The right Renal Aortic Ratio was elevated at 4.3. Of particular interest, color doppler imaging demonstrated a "mosaic" pattern extending from the distal right renal artery into the helium. The left renal artery doppler interrogation was fairly unremarkable with a PSV of $144 \mathrm{~cm} / \mathrm{sec}$ and a Renal Aortic Ratio of 1.78 (Figure 1-3).

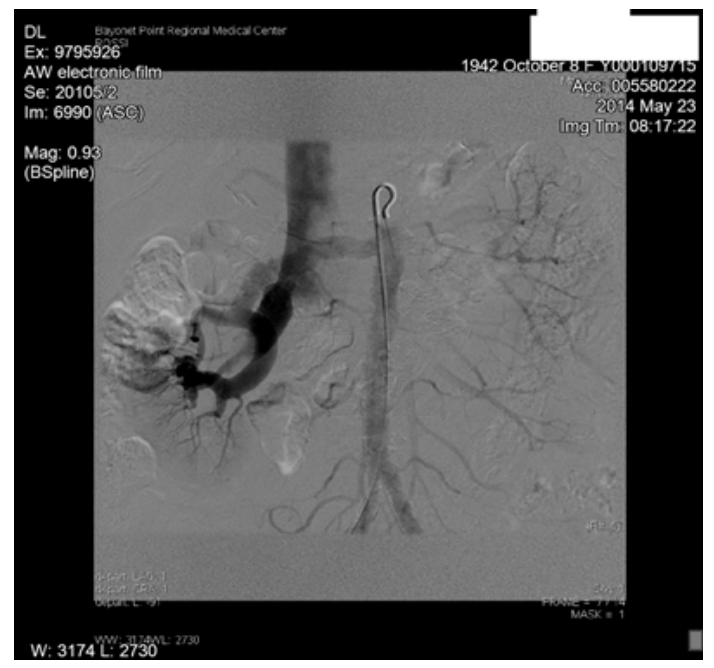

Figure I Demonstrating contrast within the IVC consistent with an A-V fistula.

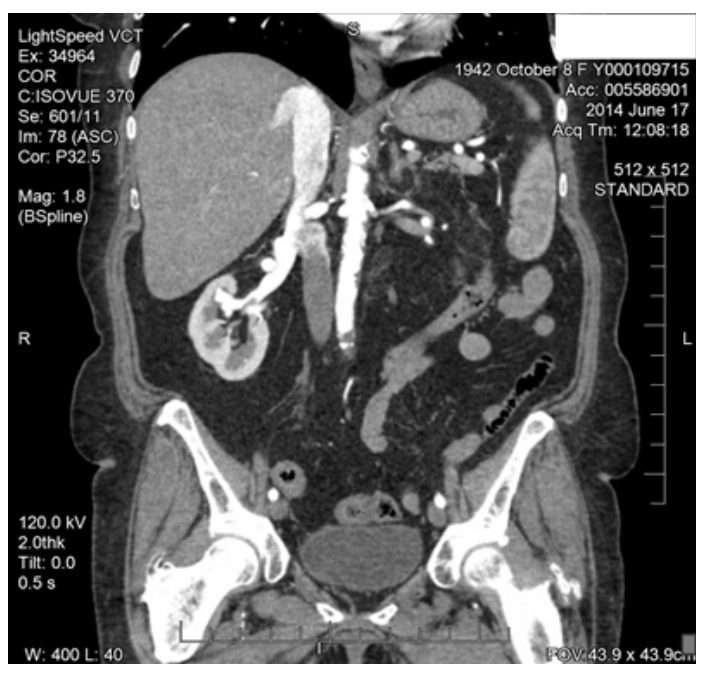

Figure 2 Demonstrating contrast within the IVC consistent with an A-V fistula.

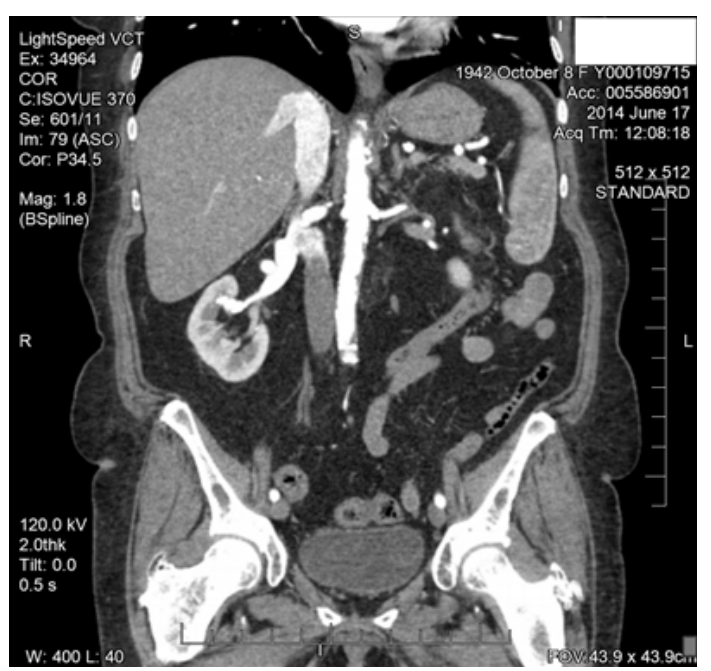

Figure 3 Demonstrating contrast within the IVC consistent with an A-V fistula.

Upon obtaining the abnormal doppler findings, our interventional cardiologist performed an abdominal angiogram with selective bilateral renal arteriography. The angiogram demonstrated diffuse 
right renal artery haustra consistent with FMH. As well, abnormal retograde filling of the right renal vein and IVC was noted, and consistent with an AV fistula within the right renal artery helium (Figure 4). The left renal artery was normal on preliminary imaging, but remained suspect. Upon IVUS (Intra Vascular Ultra Sound) imaging, a significant $(20 \mathrm{~mm})$ gradient was noted within the left distal renal artery. ${ }^{3}$ Bilateral cutting balloon angioplasties were performed and the patient was then sent to CPRU for recovery (Figure $5 \& 6$ ).

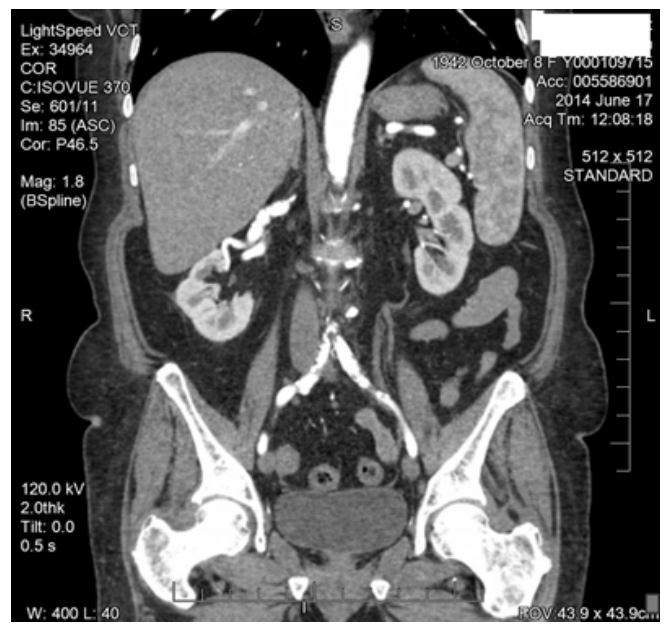

Figure 4 CTA of RRA FMD.

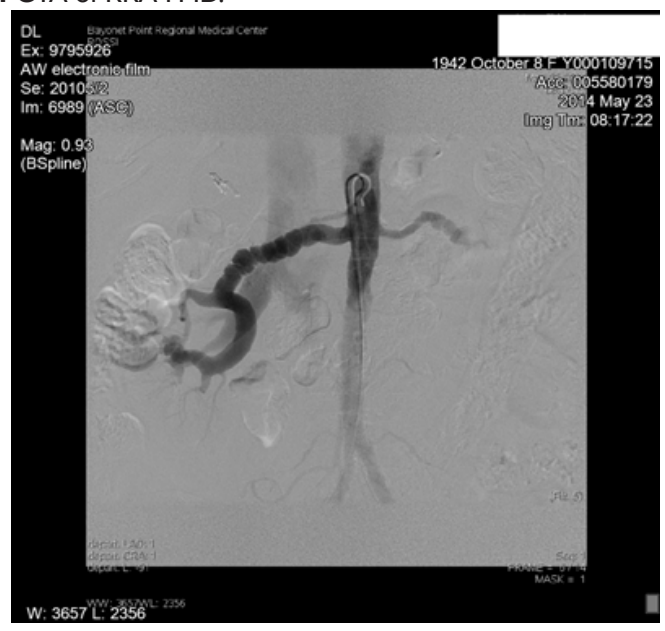

Figure 5 Angiogram images of RRA FMD.

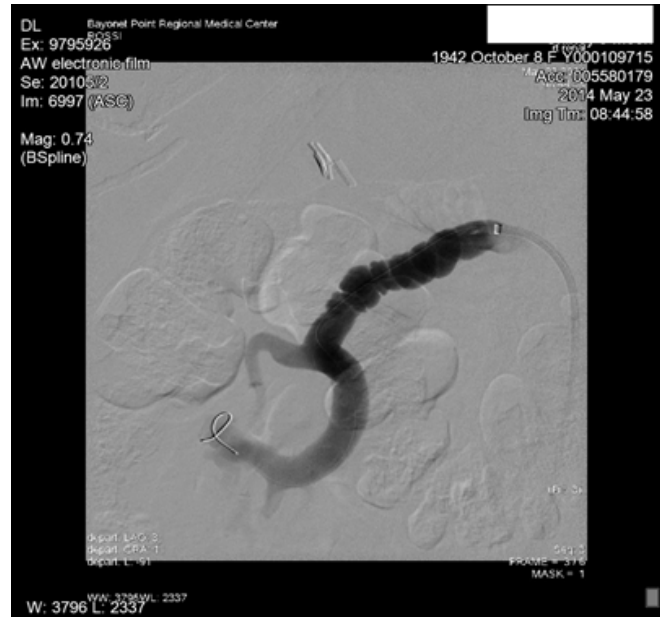

Figure 6 Angiogram images of RRA FMD.
Two months later, the patient was referred to the radiology department for additional imaging with CTA. The CTA of the abdomen verified the "banded" appearance of both renal arteries consistent with FMH. The CTA also verified the angiographic findings of a right renal artery helium AV fistula or AV malformation. Since the bilateral renal artery angioplasties, the patient's blood pressures have remained in the $130 / 70 \mathrm{~mm} \mathrm{Hg}$ range (Figure 7-9).

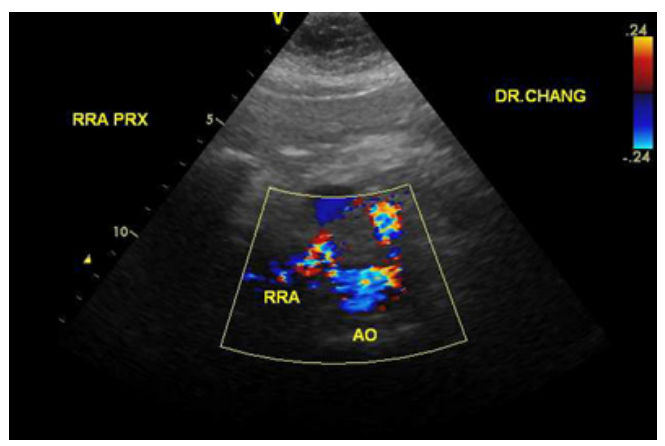

Figure 7 Color and Doppler images of RRA FMD.

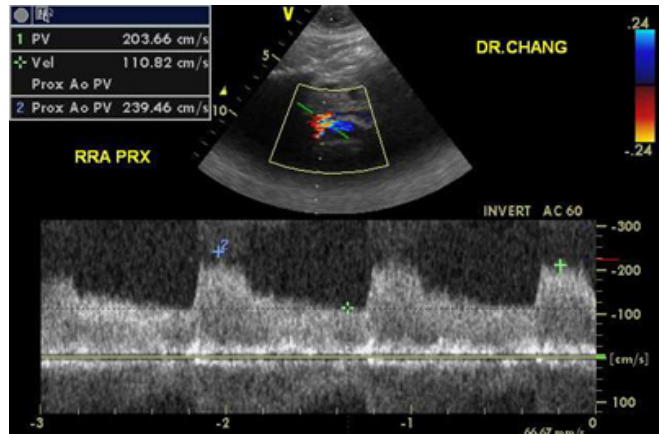

Figure 8 Color and Doppler images of RRA FMD.

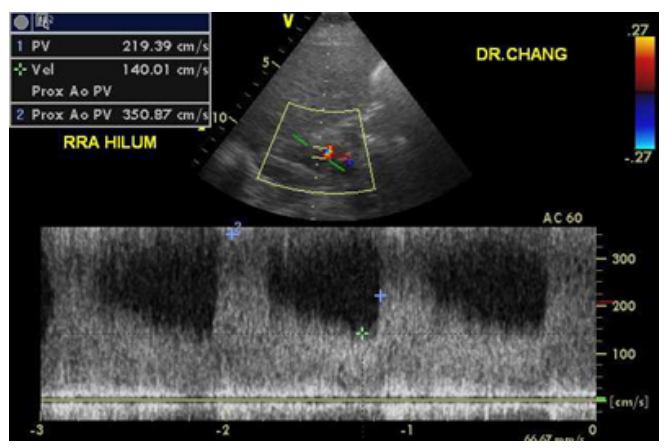

Figure 9 Color and Doppler images of RRA FMD.

\section{Conclusion}

FMH may be present in patients with uncontrolled hypertension. Typically, the patient will undergo doppler, CTA, MRA and angiography for diagnostic confirmation and intervention. FMH may be treated with various balloon angioplasty techniques and/or stenting. Often, the patient's blood pressure will be substantially lowered, if not normal status post intervention.

\section{Acknowledgments}

None.

\section{Conflicts of interest}

Author declares there are no conflicts of interest. 


\section{Funding}

None.

\section{References}

1. Tullis MJ, Caps MT, Zierler RE, et al. Blood pressure, antihypertensive medication, and atherosclerotic renal artery stenosis. Am J Kidney Dis. 1999;33(4):675-681.
2. Eugene Zierler R. Strandness's Duplex Scanning in Vascular Disorders. (4th edn), Renal Duplex Scanning, Lippincott Williams and Wilkins . 2010;23:292.

3. Olin JW, Froehlich J, Gu X, et al. The United States Registry for Fibromuscular Dysplasia: results in the First 447 Patients. Circulation. 2012;125(25):3182-3190. 